\title{
A!
}

This is an electronic reprint of the original article.

This reprint may differ from the original in pagination and typographic detail.

Odit, Mikhail; Sayanskiy, Andrey; Asadchy, Viktar; Belov, Pavel

\section{Microwave reflecting focusing metasurface based on water}

Published in:

2018 IEEE International Symposium on Antennas and Propagation \& USNC/URSI National Radio Science Meeting

DOI:

10.1109/APUSNCURSINRSM.2018.8609338

Published: 01/01/2018

Document Version

Peer reviewed version

Please cite the original version:

Odit, M., Sayanskiy, A., Asadchy, V., \& Belov, P. (2018). Microwave reflecting focusing metasurface based on water. In 2018 IEEE International Symposium on Antennas and Propagation \& USNCIURSI National Radio Science Meeting (pp. 773-774). (Digest of the IEEE Antennas and Propagation Society International Symposium). IEEE. https://doi.org/10.1109/APUSNCURSINRSM.2018.8609338

This material is protected by copyright and other intellectual property rights, and duplication or sale of all or part of any of the repository collections is not permitted, except that material may be duplicated by you for your research use or educational purposes in electronic or print form. You must obtain permission for any other use. Electronic or print copies may not be offered, whether for sale or otherwise to anyone who is not an authorised user. 
This is the accepted version of the original article published by IEEE.

(C) 2018 IEEE. Personal use of this material is permitted. Permission from IEEE must be obtained for all other uses, in any current or future media, including reprinting/republishing this material for advertising or promotional purposes, creating new collective works, for resale or redistribution to servers or lists, or reuse of any copyrighted component of this work in other works. 


\title{
Microwave reflecting focusing metasurface based on water
}

\author{
Mikhail Odit*, Andrey Sayanskiy*, Viktar Asadchy ${ }^{\dagger}$, and Pavel Belov* \\ *Department of Nanophotonics and Metamaterials, ITMO University, St. Petersburg, Russia, odit@mail.ifmo.ru \\ ${ }^{\dagger}$ Department of Electronics and Nanoengineering, Aalto University, Espoo, Finland, viktar.asadchy@aalto.fi
}

\begin{abstract}
In this work we demonstrate the first design of the focusing reflecting all-dielectric metasurface which is realized on periodically arranged bianisotropic elements made of distilled water. The metasurface was shown to demonstrate focusing capabilities of the reflected electromagnetic signal at $12 \mathrm{GHz}$ while preserving transparency outside of the operating frequency band.
\end{abstract}

\section{INTRODUCTION}

The concept of designing arbitrary material parameters passed from bulk metamaterails to metasurfaces.In comparison with bulk metamaterials they are compact and relatively simple to manufacture. Metasurfaces allow controlling the properties of the transmitted or reflected electromagnetic wave. This includes a control of a specific phase, amplitude, and polarization of electromagnetic waves [1].

Incorporating dielectric particles in the metasurface introduces new advantages such as absence of the ohmic losses and the ability to interact independently with the electric and magnetic field of the incident wave. Moreover dielectric particles with broken symmetry exhibit different types of bianisotropic behavior opening possibility to form magnetic resonant response induced by the electric field and vice versa [2]-[3]. This allows more efficient control of the phase and amplitude of the transmitted/reflected wave. At the same time, the use of dielectric particles make it possible to construct a metasurfaces which are transparent outside of the operating frequency band which is desired properties for many applications to avoid reflection or the useful signal.

Reflecting metasurfaces can be efficiently used as focusing reflectors for antennas. This metasurface can be realized with be made with metal [4] or dielectric [5] inclusions. Last years water attracts more attention as a promising dielectric material for the metasurfaces [6]-[8]. It is one of the most abundant resources on Earth which is almost free in comparison with microwave materials. Water at room temperature is has a liquid phase and takes the shape of its container allowing flexible control of its geometry [9]. Water has high permittivity at radio frequencies, $\varepsilon \approx 80$ [10], and can serve as a uniform material base for all-dielectric metasurfaces consisting of subwavelength resonators. Additionally water properties strongly depends on its temperature which makes it possible to design tunable metasurfaces with controllable properties.

It was shown that water-based metasurfaces can behave as multiband absorber [7] which can be also temperature controlled [8]. Water-based metasurface also can act as a tunable filter where tuning is realized mechanically by changing the shape of the water cells. It was shown numerically [6] and confirmed experimentally [9]. In this study we present a design off all-dielectric metasurface which provides the focusing of the reflected wave. At the same time being essentially dielectric it preserves transparency outside the operating frequency band.

\section{MetasurfaCE DESIGN}

The metasurface under study is composed of the dielectric bianisotropic particles (Fig. 1). The shape of the single particle has a form of cylinder of the height $H$ and radius $R$ with a notch inside having the depth $h$ and the radius $r$ (Fig. 1.a). The dielectric properties of the particles are the same as for distilled water at the temperature $20^{\circ}: \varepsilon=78, \mu=1$, electric conductivity $\sigma=1.59[\mathrm{~S} / \mathrm{m}]$. The period of the array is $a=10 \mathrm{~mm}$. Note that period should be less than half of the operating wavelength to avoid undesired reflection.

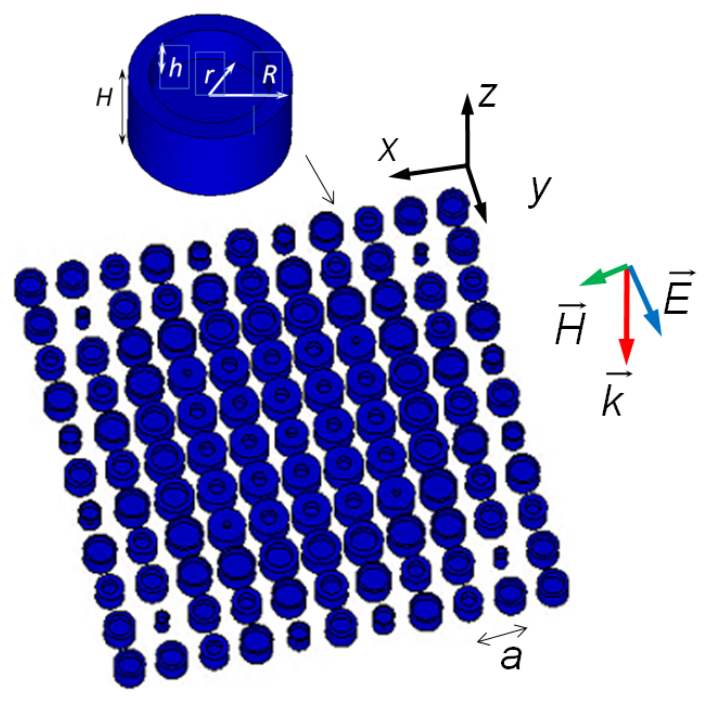

Fig. 1. The metasurface based on $11 \times 11$ high permittivity dielectric bianisotropic particles made of distilled water. The particles are arranged with a period $a$.

The metasurface is constructed in a way to provide the constructive interference of the reflected field at a focal point in front of the metasurface. We consider symmetrical reflecting metasurface with the axial symmetry. In general case the shape of the metasurface can be arbitrary depending on the 
application. The required phase distribution at the reflectarray plane can be calculated at each point using the equation:

$$
\phi\left(r_{0}\right)=\phi_{0}+\frac{2 \pi}{\lambda} \sqrt{r_{0}^{2}+F^{2}},
$$

where $\phi$ is a desired phase shift, $r_{0}$ is the distance from the lens center to the point at the surface of the lens, $F$ is focal distance, $\lambda$ is the operational wavelength and $\phi_{0}$ is an arbitrary constant phase. For the given number of the elements the phase shift is calculated for the point in the center of each resonator. The calculated phase distribution for the each element of the metasurfaces is depicted in Fig. 22 a. The phases were calculated to provide focusing of the reflected signal at the distance $F=4 \lambda$ at the frequency $f=12 \mathrm{GHz}$.

The required phase shift for each element is provided by changing the sizes of the particle. From the manufacturing point of view it is more convenient to change the inner $r$ and outer $R$ radii of the particle. The heights of the particles were fixed. First we have simulated the response of the single particle with imposed Floquet boundary conditions. The simulation was performed with the Frequency domain solver of the CST Microwave Studio. As a result the set of the complex values of the reflection coefficient were been obtain for different particle sizes. To design reflecting metasurface it is essential to provide the range of phase difference close to $2 \pi$.

Next we have selected required dimensions in order to provide phases distribution from Fig. 2/a while keeping maximum possible value of the reflection coefficient. In our design with the specified heights of $H=2.8 \mathrm{~mm}$ and $h=1.4 \mathrm{~mm}$ the radii were changed from $r=2 \mathrm{~mm}$ to $r=7 \mathrm{~mm}$ and big radii from $R=3.6 \mathrm{~mm}$ to $R=10 \mathrm{~mm}$. The difference between desired and obtained phases were less than $10^{\circ}$ while minimum amplitude of the reflection coefficient was 0.8 .

At the next step the finite size metasurface (see Fig 1) has been numerically simulated with the time integral equation solver of CST Microwave Studio. Open boundaries have been imposed from all directions and plane wave has been chosen as the excitation source.The background material was selected as air.

The performance of the designed optical metamirror is demonstrated in Fig. 2, b where simulated value ofEcomponent of the reflected incident field is depicted. The metasurface is shown in the bottom of the figure. It is seen that reflected field has a maximum at a distance $F \approx 4 \lambda$ from the metasurface. The maximum value of the reflected field amplitude is $2.2 E_{0}$, where $E_{0}$ is the amplitude of the incident field. This structure can be further optimized by proper selection of the heights and period of the elements. Thus, we have designed the focusing lens which operates at its reflection regime and it is composed of all-dielectric materials.

\section{CONCLUSION}

We have introduced a new type of the all-dielectric metamaterial surface which based on dielectric inclusions made of

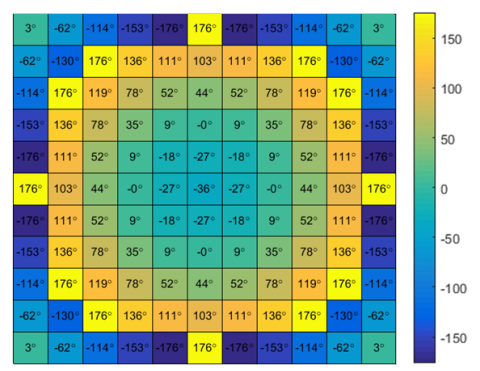

(a)

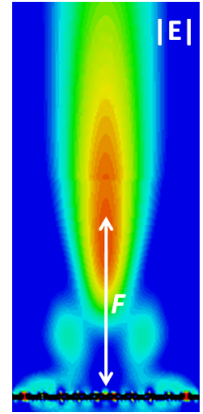

(b)
Fig. 2. (a) Required phase distribution for the metasurface consisting of $11 \times 11$ bianisotropic elements. (b) Maximum value of the electric field reflected from the metasurface. ]

distilled water. This type of metasurfaces can be used to construct large-scale and low-cost reflecting array which remains transparent outside of the operating frequency band. Moreover this transparency also preserves at optical frequencies being remarkable feature from the point of view of aesthetics or military design. The results of numerical simulations of the metasurface performance revealed the possibility of the reflected electromagnetic wave focusing at the frequency of $12 \mathrm{GHz}$. This frequency can be easily changed by the proper design of the metasurface or even tuned by different ways using unique properties of water.

\section{ACKNOWLEDGEMENT}

This work was supported by the the Russian Science Foundation (Project No. 17-19-01731).

\section{REFERENCES}

[1] Stanislav B.Glybovski, Sergei A. Tretyakov, Pavel A.Belov, Yuri S.Kivshar, Constantin R.Simovski, "Metasurfaces: From microwaves to visible," Phys. Rep., vol. 634, pp. 1-72, May 2016.

[2] Rasoul Alaee, Mohammad Albooyeh, Aso Rahimzadegan, Mohammad S. Mirmoosa, Yuri S. Kivshar, and Carsten Rockstuhl, "All-dielectric reciprocal bianisotropic nanoparticles," Phys. Rev. B, 92, 245130, December 2015.

[3] M. Odit, P. Kapitanova, P. Belov, R. Alaee, C. Rockstuhl and Y. S Kivshar, "Experimental realization of all-dielectric bianisotropic metasurfaces,“ Appl. Phys. Lett. 108, 221903, 2016.

[4] B.-C. Lin, G.-M. Wang, T. Cai, "Transmissive focusing meta-surface with nearly 100\% efficiency,“ Appl. Phys. 123:630, 2017.

[5] V. Asadchy, M. Albooyeh, and S. Tretyakov, "Optical metamirror: alldielectric frequency-selective mirror with fully controllable reflection phase," Journal of the Opt. Soc. of America B Vol. 33, Issue 2, pp. A16-A20, 2016.

[6] A. Andryieuski, S.M. Kuznetsova, S.V. Zhukovsky, Y.S. Kivshar, A.V Lavrinenko, "Water: Promising Opportunities For Tunable All-dielectric Electromagnetic Metamaterials,“Sci. Rep., 5, 13535, 2015.

[7] W. Zhu, I. Rukhlenko, F. Xiao, C. He, J. Geng, X. Liang, M. Premaratne, and R. Jin,"Multiband coherent perfect absorption in a water-based metasurface," Opt. Express 25, 15737-15745, 2017.

[8] Y. Pang, J. Wang, Q. Cheng, et al.,"Thermally tunable water-substrate broadband metamaterial absorbers, “'Appl. Phys. Lett., 110, 104103, 2017.

[9] M. Odit, P. Kapitanova, A. Andryieuski, P. Belov, and A. V. Lavrinenko,"Experimental demonstration of water based tunable metasurface," Appl. Phys. Lett., 109, 011901, 2016.

[10] W. Ellison, "Permittivity of pure water, at standard atmospheric pressure, over the frequency range $025 \mathrm{THz}$ and the temperature range $0100 \mathrm{C}$, “ $J$. Phys. Chem. Ref., Data 36, 118, 2007. 